Proceedings

\title{
Live Tracking Biofunctionalization and Label-Free Protein Detection Performed by a Nanophotonic Biosensor ${ }^{+}$
}

\author{
Jad Sabek ${ }^{1, *}$, Luis Torrijos-Morán ${ }^{1}$, Zeneida Díaz-Betancor ${ }^{2}$, María José Bañuls-Polo ${ }^{2}$, \\ Ángel Maquieira-Catalá ${ }^{2}$ and Jaime García-Rupérez ${ }^{1, *}$ \\ 1 Nanophotonics Technology Center, Universitat Politècnica de València. Camí de Vera s/n, Valencia, Spain; \\ luitorm2@ntc.upv.es \\ 2 Departamento de Química, Instituto Interuniversitario de Investigación y de Reconocimiento Molecular y \\ Desarrollo Tecnológico IDM, Universitat Politècnica de València, Camí de Vera s/n, Valencia, Spain; \\ zediabe@upvnet.upv.es (Z.D.B.); mbpolo@upvnet.upv.es (M.J.B.P.); amaquieira@qim.upv.es (Á.M.C.) \\ * Correspondence: jasa@ntc.upv.es (J.S.); jaigarru@ntc.upv.es (J.G.-R.) \\ + Presented at the 5th International Electronic Conference on Sensors and Applications, 15-30 November \\ 2018; Available online: https://ecsa-5.sciforum.net.
}

Published: 14 November 2018

\begin{abstract}
A label-free biosensor based on silicon-on-insulator (SOI) photonic bandgap (PBG) structures is performed for specific protein detection. First, the SOI sensing surface is functionalized using triethoxyvinylsilane (TEVS) organosilane. Then, a UV light photocatalyzed immobilization of polyclonal half anti-bovine serum albumin (haBSA) antibodies is performed. Finally, a direct detection of target BSA antigen is carried out. Both the immobilization and the detection steps are monitored by making a continuous tracking of the PBG edge shift. In order to confirm the recognition of the antigen by the immobilized antibody, a fluorophore-labelled secondary antibody was flowed at the end of the experiment in order to perform a confirmation fluorescence test after the photonic detection.
\end{abstract}

Keywords: photonic sensor; photonic bandgap; silicon on insulator; biofunctionalization; photocatalysis; half antibodies

\section{Introduction}

During the last few years, a significant progress has been made in the development of photonicbased biosensing devices. These devices offer several advantages over the conventional methodologies such as PCR or ELISA, which are expensive, bulky, time consuming, and lab centralized. Evanescent wave based photonic biosensors can provide a high sensitivity detection in a label-free scheme, with a high multiplexing potential for parallelized analysis, making them suitable for the development of high-performance lab-on-a-chip devices [1]. Additionally, they are typically based on standard silicon technology fabrication, allowing affordable mass production and miniaturization [2].

However, in order to achieve high sensitivity, high selectivity and accuracy in a biosensing platform, a trustworthy functionalization protocol for the immobilization of the receptors on the sensing structure must be available. This would allow analyzing chemical and biological samples without any pretreatment (for example, labelling or filtering). Among the different procedures for the biofunctionalization of sensing structures, covalent strategies for the immobilization of bioreceptors provide several advantages in terms of non-specific interactions restriction and 
thickness reduction of the recognition layer, compared to other simpler strategies based on physical adsorption.

Here, we report our work towards the development of high sensitivity and selective photonicbased biosensors. We have used photonic bandgap (PBG) structures as sensing elements since they have emerged as one of the most sensitive photonic configurations for a label-free detection [3]. In these structures, the introduction of a periodic modulation in the refractive index of the photonic structure gives rise to the appearance of a rejected spectral band, referred to as the PBG [4], whose position will depend on the refractive index of the surrounding medium with high sensitivity. In order to provide selectivity to the PBG sensing structures, antibodies specific to Bovine Serum Albumin (BSA) have been immobilized on the sensor's surface. However, instead of using whole antibodies, half BSA antibodies (haBSA) obtained using tris(2-carboxyethyl)phosphine (TCEP) reduction have been used to obtain a higher surface coverage density, a lower thickness of the recognition layer and to have proper orientation of the antibodies binding sites. The linkage of the haBSA antibodies to the sensors has been performed via the thiol moieties available after the TCEP reduction by covering the surface with vinyl groups using triethoxyvinyl-silane (TEVS) [5]. Upon the illumination of the sensor with UV light, the reaction between the thiol and the vinyl groups takes place and the covalent immobilization of the haBSA antibodies is produced leading to a thioeter for the covalent immobilization. Finally, the sample containing the target BSA antigens was flowed over the PBG sensing structure in order to be specifically recognized by the haBSA antibodies immobilized on the sensor's surface. Both the haBSA antibodies immobilization and the BSA recognition steps have been monitored continuously using the PBG sensor, which has allowed us to experimentally demonstrate the binding of the haBSA antibodies to the surface only upon the excitation with UV light and the subsequent recognition of the BSA targets by the biofunctionalized photonic biosensor.

\section{Materials and Methods}

Figure 1 shows the pictures of the fabricated photonic chip containing the PBG sensing structures. It has been created in a silicon-on-insulator (SOI) substrate using e-beam lithography with an acceleration voltage of $100 \mathrm{keV}$ and inductively coupled plasma etching of the top silicon layer. The structural parameters of the PBG sensing structures are height $\mathrm{h}=220 \mathrm{~nm}$, waveguide width $\mathrm{w}$ $=450 \mathrm{~nm}$, period $\mathrm{a}=380 \mathrm{~nm}$, transversal elements length $\mathrm{we}_{\mathrm{e}}=1500 \mathrm{~nm}$ and transversal elements widths ranging from $\mathrm{w}_{\mathrm{i}}=80 \mathrm{~nm}$ to $\mathrm{W}_{\mathrm{i}}=140 \mathrm{~nm}$. These structural parameters provide PBG edges located in the wavelength range between $1500 \mathrm{~nm}$ and $1600 \mathrm{~nm}$, where our experimental characterization equipment operates. The chip is accessed at the input and the output via $70 \mathrm{~nm}$-deep shallows etch 1D grating couplers [6].

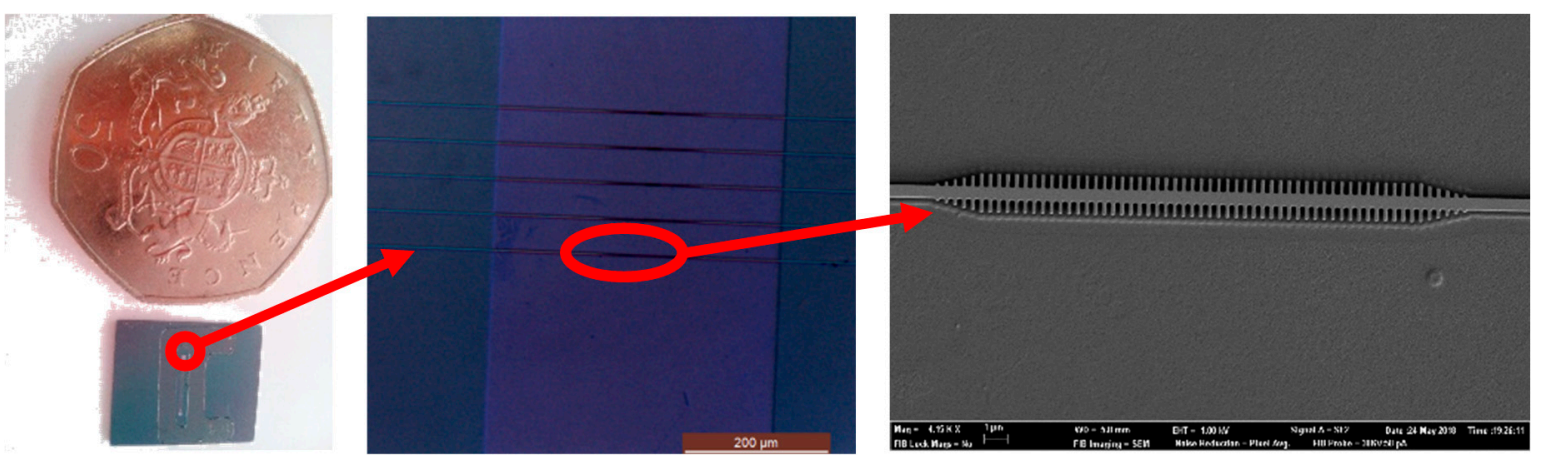

Figure 1. (Left) Image of the photonic chip fabricated in Silicon On Insulator (SOI) technology. The circle depicts the position of each photonic bandgap (PBG) sensors groups. (Center) Microscope image of a PBG sensors group. The circle depicts an individual PBG sensing structure. (Right) Scanning Electron Microscope (SEM) image of a fabricated PBG sensing structure.

To obtain a vinyl-terminated monolayer on the surface of the SOI PBG sensing structures, where the haBSA antibodies will be immobilized via their thiol moieties using a light-assisted thiolene coupling (TEC) reaction, the photonic chip is silanized using triethoxyvinyl-silane (TEVS). The steps 
of the silanization process used in this work are depicted in Figure 2. Typically, the silanization process is carried out using an organic anhydrous solvent as a carrier for the organosilane, such as toluene [7]. However, the use of organic solvents can lead to several problems such as vertical polymerization, which produces a thicker silane layer that will increase the distance between the photonic sensors and the target analytes that will reduce sensitivity, and generate organic waste [810]. Taking these problems into account, we have performed the silanization process using water as a carrier for the TEVS organosilane. Despite the solubility of TEVS in water not being complete, the exchange of its triethoxy groups with $\mathrm{OH}$ groups from the water makes it stable. In order to have an optimal triethoxy $\leftrightarrow \mathrm{OH}$ exchange ratio and to finally obtain a compact vinyl monolayer on the surface, the $\mathrm{pH}$ of the solution is adjusted to 8 using $\mathrm{KOH}$ or $\mathrm{NaOH}$. Therefore, the final silanization process consisted on immersing the SOI chip in 1\% TEVS in MilliQ water (pH adjusted to 8 by adding $100 \mu \mathrm{L}$ from $1 \mathrm{M} \mathrm{KOH}$ mother dissolution) during $1 \mathrm{~h}$ and finally curing it at $110^{\circ} \mathrm{C}$ during $1 \mathrm{~h}$ for condensation and water excess evaporation. Note that, before performing the silanization process, the SOI chip was cleaned in a piranha solution $\left(\mathrm{H}_{2} \mathrm{SO}_{4} / \mathrm{H}_{2} \mathrm{O}_{2}: 1 / 3\right)$ for $20 \mathrm{~min}$ and then activated using $\mathrm{O}_{2}$ plasma for $10 \mathrm{~min}$.

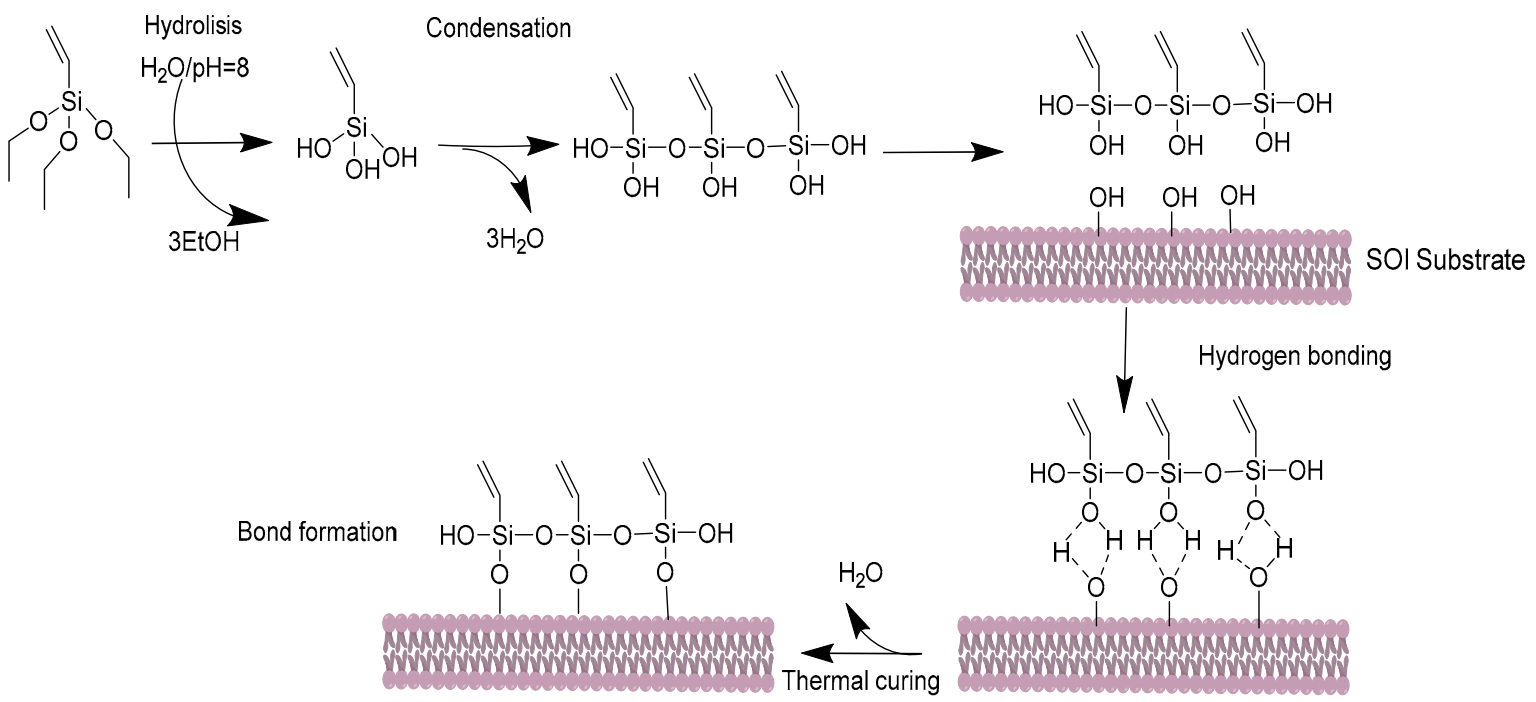

Figure 2. Steps of the silanization process used to create a vinyl-terminated monolayer on the surface of the SOI sensing structures.

In order to covalently immobilize the BSA antibodies over the sensor surface using the TEC reaction, they are first divided using the tris(2-carboxyethyl)phosphine (TCEP) reduction method, as described in [5]. After the TCEP reduction, thiol moieties will be available on the resulting haBSA antibodies for their immobilization over the vinyl-terminated surface by the mean of UV light (254 $\mathrm{nm})$ photocatalysis. Figure 3 schematically shows the haBSA immobilization scheme employed.
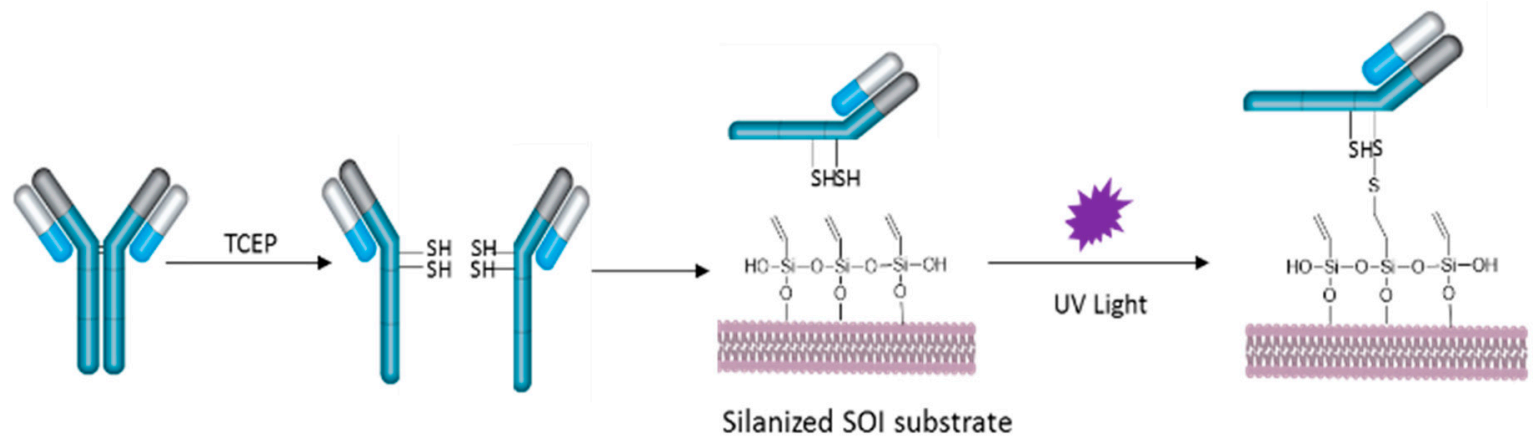

Figure 3. Schematic representation of the process used for the covalent immobilization of the haBSA antibodies on the PBG sensors surface. 
Finally, once the haBSA bioreceptors are immobilized on the surface, the target BSA antigen was flowed over the chip to be detected by the PBG sensing structures.

\section{Results and Discussion}

Figure 4 shows the images of the characterization of the chip carried out after performing the TEVS silanization process described above. The water contact angle (WCA) test indicates an increase of the hidrophobicity of the surface, which confirms the coverage with vinyl groups. On the other hand, the Atomic Force Microscope (AFM) characterization of the surface shows a very low roughness, also confirming the proper creation of the organosilane layer. Additionally, ellipsometry measurements were carried out, providing a TEVS layer thickness of $0.33 \pm 0.03 \mathrm{~nm}$, confirming again the creation of a compact organosilane monolayer.

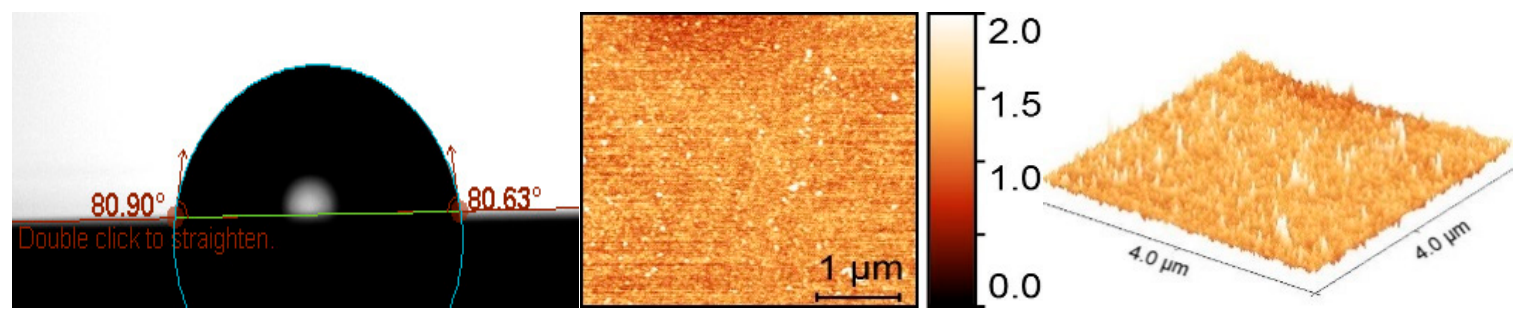

Figure 4. (Left) WCA test confirming the hydrophobicity increase of the surface. (Right) AFM topography characterization of the silanized SOI surface.

The silanized chip is then assembled with a PDMS-based microfluidic cell used to flow the reagents over the photonic chip while its sensing response is continuously monitored. The optical characterization set up used for the interrogation of the photonic sensing chip mainly consists on a continuous sweep tunable laser. This is synchronized with an infrared camera to acquire the spectral response of the different photonic structures within the chip and to track their spectral shift during the sensing experiments.

Figure 5 shows a representative spectral evolution of the PBG edge for one of the sensing structures within the chip during the haBSA immobilization step. Initially, Phosphate Buffered Saline (PBS) $1 \times$ was flowed over the chip to obtain the initial baseline. Then, the solution containing the reduced haBSA $(200 \mu \mathrm{g} / \mathrm{mL}$ in PBS $1 \times)$ was flowed. As can be observed in Figure 5, no photonic sensing response was obtained, despite both the surface vinyl groups and the thiol moieties of the haBSA antibodies being present. It is not until the photonic chip is irradiated with UV light that the vinyl-thiol reaction is photocatalyzed and the haBSA antibodies are immobilized on the sensor surface, what is translated into a shift of the PBG position. Finally, PBS $1 \times$ buffer is flowed again in order to determine the net spectral shift and to remove any excess of haBSA antibodies.

Finally, BSA $1 \mu \mathrm{g} / \mathrm{mL}$ in PBS-T (PBS $+0.01 \%$ Tween 20) is flowed over the biofunctionalized sensing surface to characterize its specific recognition by the immobilized haBSA antibodies (the buffer is changed from PBS to PBS-T to prevent non-specific interactions and BSA adsorption). Figure 6(Left) depicts representative results of this experiment, where we can see that the target BSA is properly detected by the PBG sensing structure. In order to corroborate the haBSA-BSA recognition, a fluorophore-labelled secondary BSA antibody was then flowed over the photonic chip for fluorescence characterization (see Figure 6(Right)). 


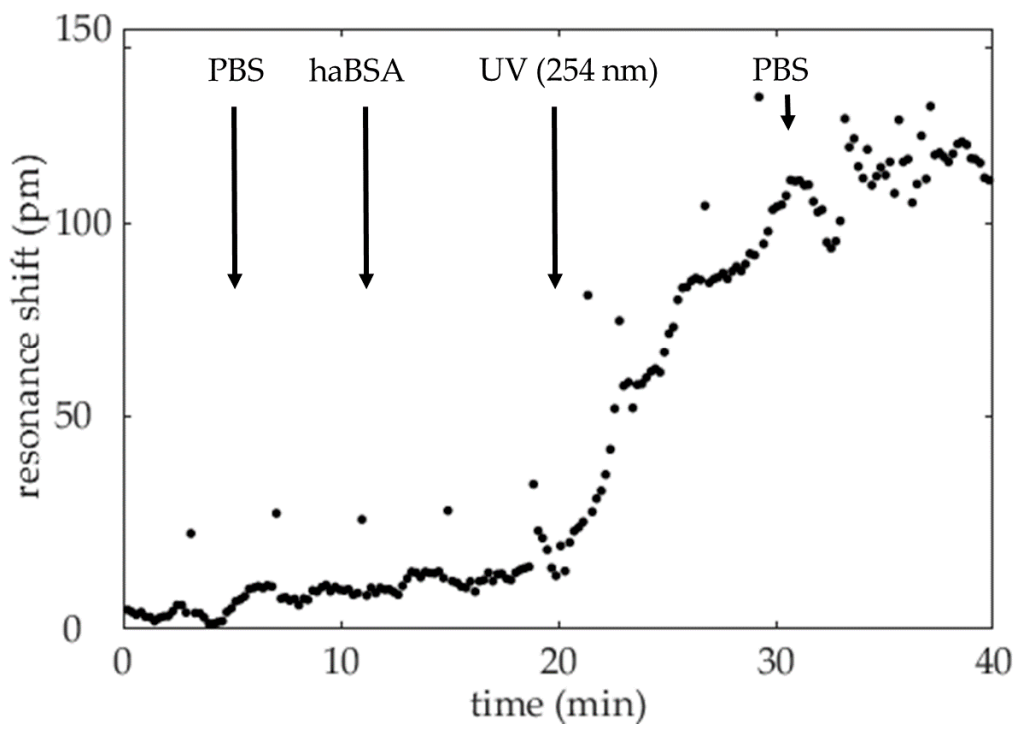

Figure 5. Temporal evolution of the PBG spectral shift during the light-assisted haBSA immobilization process. haBSA are injected at 10-minutes and UV light source is switched on at 20-minutes.
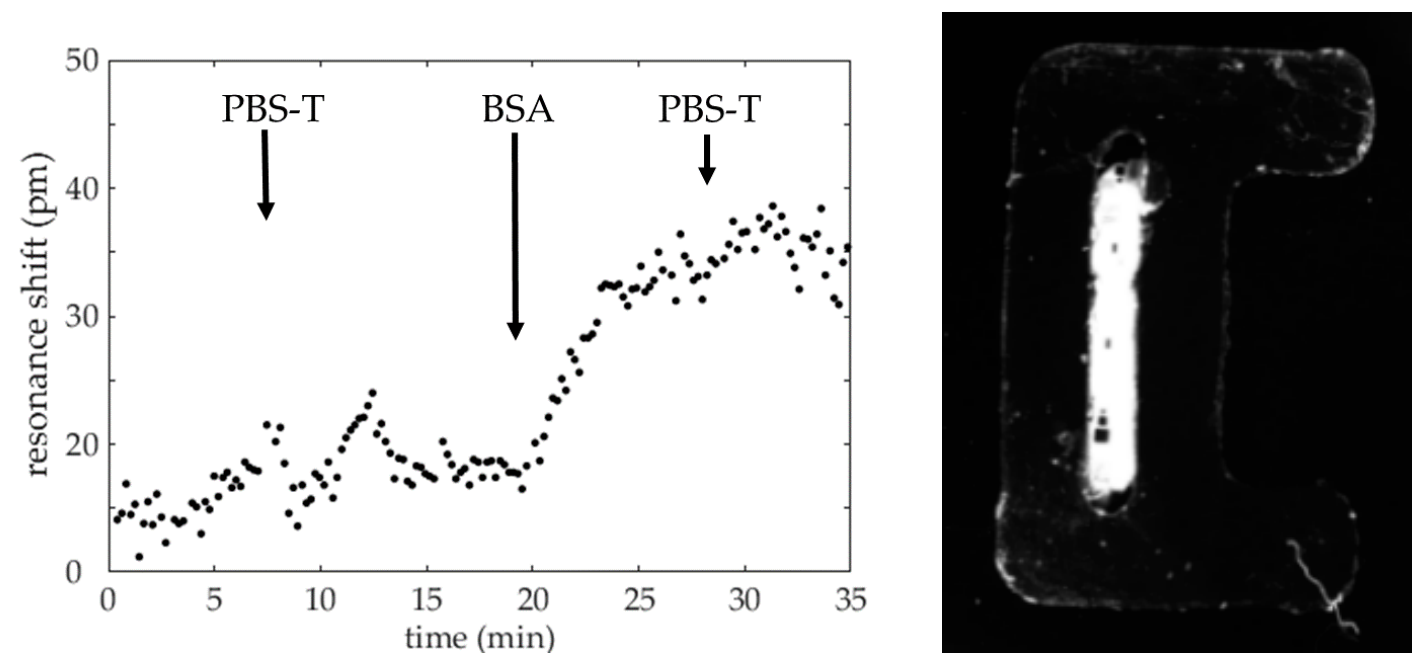

Figure 6. (Left) Temporal evolution of the PBG spectral shift during the BSA detection experiment. (Right) Fluorescence test was carried out after binding a fluorophore-labelled secondary aBSA antibody to the recognized BSA target.

\section{Conclusions}

In this work, the development of a PBG photonic biosensor for the label-free detection of proteins is presented. To our knowledge, this is the first time that a photonic sensing structure biofunctionalized with half antibodies as recognition layer is reported. The use of half antibodies provides several important benefits for the development of higher sensitivity biosensors, such as a higher surface coverage density, a lower thickness of the recognition layer and a proper orientation of the antibodies binding sites. Additionally, the light-assisted immobilization of the haBSA antibodies on the sensing surface using TEC biofunctionalization has been tracked in-live, confirming that the reaction between the vinyl groups on the surface and the haBSA thiol moieties are only produced upon UV light photocatalysis.

Acknowledgments: This work was supported by Horizon 2020 Framework Program of the European Union under the project H2020-PHC-634013 (PHOCNOSIS).

Conflicts of Interest: The authors declare no conflict of interest. 


\section{References}

1. Jokerst, J.V.; Jacobson, J.W.; Bhagwandin, B.D.; Floriano, P.N.; Christodoulides, N.; McDevitt, J.T. Programmable nano-bio-chip sensors: Analytical meets clinical. Anal. Chem 2010, 82, 1571, doi:10.1021/ac901743u.

2. Dutta, H.S.; Pal, S. Design of a highly sensitive photonic crystal waveguide platform for refractive index based biosensing. Opt. Quantum Electron. 2013, 45, 907-917, doi:10.1007/s11082-013-9697-x.

3. Wang, X.; Flueckiger, J.; Schmidt, S.; Grist, S.; Fard, S.T.; Kirk, J.; Doerfler, M.; Cheung, KC.; Ratner, DM.; Chrostowski, L. A silicon photonic biosensor using phase-shifted Bragg gratings in slot waveguide. J. Biophotonics 2013, 6, 821-828, doi:10.1002/jbio.201300012.

4. Povinelli, M.; Johnson, S.; Joannopoulos, J. Slow-light, band-edge waveguides for tunable time delays. Opt. Express 2005, 13, 7145-7159, doi:10.1364/OPEX.13.007145.

5. Alonso, R.; Jiménez-Meneses, P.; García-Rupérez, J.; Bañuls, M.J.; Maquieira, Á. Thiol-ene click chemistry towards easy microarraying of half-antibodies. Chem. Commun. 2018, 54, 6144-6147, doi:10.1039/c8cc01369a.

6. García, J.; Sanchis, P.; Martínez, A.; Martí, J. 1D periodic structures for slow-wave induced non-linearity enhancement. Opt. Express 2008, 16, 3146-3160, doi:10.1364/OE.16.003146.

7. Haensch, C.; Hoeppener, S.; Schubert, U.S. chemical modification of self-assembled silane based monolayers by surface reactions. Chem. Soc. Rev. 2010, 39, 2323-2334, doi:10.1039/B920491A.

8. Kim, J.; Seidler, P.; Wan, L.S.; Fill, C. Formation, structure, and reactivity of amino-terminated organic films on silicon substrates. J. Colloid Interface Sci. 2009, 329, 114-119, doi:10.1016/j.jcis.2008.09.031.

9. Phaner-Goutorbe, M.; Dugas, V.; Chevolot, Y.; Souteyrand, E. Silanization of silica and glass slides for DNA microarrays by impregnation and gas phase protocols: A comparative study. Mater. Sci. Eng. C 2011, 31, 384-390, doi:10.1016/j.msec.2010.10.016.

10. Wang, A.; Tang, H.; Cao, T.; Salley, S.O.; Ng, K.Y. In vitro stability study of organosilane self-assemble monolayers and multilayers. J. Colloid Interface Sci. 2005, 291, 438-447, doi:10.1016/j.jcis.2005.05.008.

(C) 2018 by the authors. Licensee MDPI, Basel, Switzerland. This article is an open access article distributed under the terms and conditions of the Creative Commons Attribution (CC BY) license (http://creativecommons.org/licenses/by/4.0/). 